\title{
Sieben Briefe Albrecht von Hallers an Johannes Gessner
}

\author{
Mitgeteilt von Erich Hintzsche
}

Im Jahre 1923 hat H. E. Sigerist 549 Briefe Albreght von Hallers an JoHANNes Gessner publiziert ${ }^{1}$ und damit der Forschung ein wertvolles Quellenmaterial für wissenschaftsgeschichtliche Untersuchungen zugänglich gemacht. Gegenüber dem Umfang dieser außergewöhnlich inhaltsreichen Sammlung mögen die im folgenden veröffentlichten 7 Briefe HALLERS an Gessner unbedeutend erscheinen. Mehrere davon enthalten jedoch bemerkenswerte Angaben zur Biographie Hallers, so daß ihre Bekanntgabe Interesse finden dürfte. $\mathrm{Da} \beta$ die Publikation gerade in einer H.E. SigERIST gewidmeten Gabe erfolgen kann, ist mir eine besondere Freude; mag er sie als ein Zeichen des Dankes für viele Anregung aus geschriebenem und gesprochenem Wort entgegennehmen und gleichzeitig daraus erkennen, daß er in einem seiner frühesten Arbeitsgebiete, der HALLER-Forschung, immer wieder Nacheiferer findet.

Von den der Stadt- und Hochschulbibliothek Bern gehörenden Briefen Albrecht von Hallers an Johannes Gessner ${ }^{2}$ stammen 3 sicher aus dem Antiquariatshandel. Der früheste ist datiert: Königsfelden, den 19. Juni 1729; er trägt die Zuwachsnummer 1920/688 und wurde vom Schiller-Verlag Vitznau am 29. März 1920 erworben. Ein weiteres Blatt mit dem Datum des 24. März 1747, jedoch ohne Ortsangabe, hat die Zuwachsnummer 1937/3000, es wurde von H. Margus in La Tour-de-Peilz zusammen mit 5 weiteren Briefen Hallers am 12. November 1937 erstanden. Der dem Datum nach späteste Brief ist am 19. April 1757 aus Bern geschrieben; nach der Zuwachsnummer 1936/1882 wurde er von H. Hinterberger in Wien am 20. Juni 1936 angekauft. Wann diese Briefe - vermutlich durch einen Autographenjäger - der Sammlung Gessners entnommen wurden und auf welche Weise sie in den Handel gekommen sind, wird kaum je zu ermitteln sein.

Von den restlichen 4 Briefen, deren Ankaufszeit nicht mehr festzustellen ist, waren 3 versiegelt, auch tragen sie auf der Adresse die üblichen postalischen Vermerke. Damit ist auszuschließen, daß es sich etwa um Konzepte handelt, die mit anderem HaLler-Nachlaß in Bern geblieben wären. Das letzte Blatt, ein Brief in Kleinquart aus Bern vom 12. Mai 1743, war

${ }^{1}$ Abh. Kgl. Ges. Wiss. Göttingen Math.-phys. Kl.N.F. XI, 2. 1923.

${ }^{2}$ Signatur: Mss. Haller XVIII/78. 
gefaltet, ist aber nicht gesiegelt, ferner fehlt eine Adresse; vermutlich hat es einen anderen Sendung beigelegen. Beachtenswert erscheint mir an diesem der links oben neben dem Briefkopf befindliche kleine Prägestempel «Rob Cole» (zusammenhängend in lateinischen Buchstaben geschrieben), wahrscheinlich der Name eines früheren Besitzers. Alles in allem genommen kann es keinem Zweifel unterliegen, daß die 7 Briefe echt sind und wirklich dem Gedankenaustausch der Freunde gedient haben.

Zufälligerweise lassen sich die 7 Briefe drei Epochen in HaLlers Leben zuordnen: 2 stammen aus dem Jahre 1729, der Zeit von HALlers Rückkehr in die Heimat nach Abschluß seiner Studien; 4 fallen in die Jahre 1741-1747, also in die Göttinger Zeit, wovon jedoch einer bei Gelegenheit eines Ferienaufenthaltes 1745 in Bern geschrieben wurde; der letzte aus dem Jahr 1757 ist offenbar nur ein Teilstück, da der Briefkopf fehlt; er gehört in die Zeit von Hallers Tätigkeit als Rathausammann in Bern. Alle Briefe sind im folgenden in vollem Wortlaut wiedergegeben, wobei durch Angabe der Nummern vermerkt ist, an welchem Ort der Sigeristschen Publikation die Briefe einzuschalten sind. Erläuterungen zum Text stehen in den Anmerkungen, einige zum Teil durch Korrekturen unlesbare Worte sind durch Punkte markiert, aufgelöste Abkürzungen sind in eckige Klammern eingeschlossen. Die beigefügten Inhaltsangaben heben im wesentlichen nur biographisch interessante oder für Hallers Persönlichkeit wichtige Sätze hervor. Wegen der vielen Notizen, die vom Ankauf und Tausch von Büchern sowie von literarischen Neuigkeiten und dergleichen handeln, sei auf den lateinischen Text und auf die Anmerkungen verwiesen.

1. Brief, einzuschalten bei Sigerist (1923) zwischen Nr. 1 und 2.

Viro doctissimo J. Gesnero/M(edicinae) Cand(idato)/S.p.d./Albertus HALler.

Jam ante octiduum, cum morbo forsan diuturniori me detineri perciperem, per D. Passavantium ${ }^{3}$ Te exoravi ut libros meos et sceletos suis cistis ordinare velles. Quod officium ab amicitia Tua spero. Posset vero cista major integre claudi, pannis etiam et stramine obduci. Sceletus Argentinensis ${ }^{4}$ itidem suam cistulam excipi et libri, qui forsan in altera nimie sunt, tum

${ }^{3}$ Haller hatte in Basel «in der hohen Sommerlust» bei Dr. Cluaude Passavant (1680 bis 1743) gewohnt und sich mit dessen Söhnen angefreundet (Hintzsche, Berner Beitr. Gesch. Med. u. Naturw. 1, 41, Bern 1942).

${ }^{4}$ Am 20. April 1729 erhielt Haller ein Skelett aus Straßburg für 62 lb. (Hintzsche, l.c. $43)$. 
et capita Parisiensia hac una includi, totumque noc $^{\circ}$ iterum stramme pannisque obvolvi. Tertia cista sceleto novo destinata est, quem forte nondum perfecit D. Mieg ${ }^{6}$. Si Tibi obtingat meo nomine eum saluta et ut perfici curet insta, cum unica forte nocte Basileae conmorari possim. Caetera omnia relinquite quae ipse digeram, tertiana. Caeterum laboro et exquisita quidem. Sede declinat(a) paroxysmi aeres erant, et deliria octo horarum passum sum. Salute amicos meos StÄhelinum ${ }^{7}$, KönIGIUM ${ }^{8}$ et alios tum et Cl. D. Bernoulli' ${ }^{9}$ Vale et me ama. Königsfeldae 19. die Jun. 1729. Saluta m(ihi ?) Passavantios fratres.

Haller scheint Basel Ende Mai 1729 verlassen zu haben, wenigstens datiert die letzte Eintragung in seinem dort geführten Tagebuch vom 29. Mai $1729^{10}$. Er ist jedoch damals nicht direkt nach Bern zurückgekehrt, sondern besuchte seine Tante CATHERina EngeL in Königsfelden bei Brugg, die mit dem dort amtierenden Hofschreiber SAmuel Rodt (1690-1766) verheiratet war. Während dieses Besuches erkrankte HALLER an einem Tertianfieber, wie Sigerist schon berichtete. Die in diesem Brief vom Freunde erbetene Hilfeleistung diente der Vorbereitung des endgültigen Umzuges nach Bern. In dem kurzen Satz über gutes Gelingen seiner Arbeit dürfen wir wohl einen Hinweis auf das Fortschreiten des Gedichtes «Gedanken über Vernunft, Aberglauben und Unglauben» sehen, das Prof. StäHelin, einem Basler Freunde Hallers, gewidmet ist.

2. Brief, einzuschalten bei Sigerist (1923) zwischen Nr. 2 und 3.

Amico Singulari/Viro Doctissimo Clarissimo/Јон. Gesnero/s. p. d./ Alb. Haller.

${ }^{5}$ Offenbar das Skelett einer der Leichen, an denen HALLER während des Winters 1728/29 anatomische Demonstrationen gehalten hat; über diese vgl. E. Hintzsche, Z. Anat u. Entwicklungsgesch. Bd. 111, 1941, und WERNER KoLB, Geschichte des anatomischen Unterrichtes an der Universität zu Basel, S. 171-182, Basel 1951.

6 Johann Rudolf Mieg (1694-1733) war als Professor der Anatomie und Botanik HaLLERs Lehrer in Basel.

7 Benedikt Stähelin (1695-1750), seit 1727 Professor der Physik in Basel, einer der engsten Freunde HaLlers, dem er die Anregung zur Beschäftigung mit der englischen Dichtkunst verdankt.

8 Emanuel König jun. (1698-1752), später Professor für Anatomie und Botanik, dann für theoretische Medizin, gehörte gleichfalls zu HaLlers Freundeskreis in Basel.

9 Johann Bernoulli (1667-1748) war Hallers Lehrer der Mathematik in Basel während des Sommersemesters 1728.

10 Hintzsche, l. c. 43. 
Amicissimas Tuas cum additis variis donariis recte adcepi eam animi promtitudinem agnoscens, qua inter amicos meos semper eminuisti. Omnia aptissima sunt. Manuscripta ${ }^{11}$ simul omnia remittam proxima septimana. Serici libram eodem pretio coemtam mihi remitti cura, sic tamen ut color sit ex coeruleo albicans, blau-weiß, blanc d'azur. Pretium vel Tibi, vel alicui ut Tibi reddatur... (Korrektur) promte curabo.

Quid hic acturus sim fere nescio, adeo me absorbet nugasorum negotionem turba. Amici, cognati, patroni, quos omnes urbe nuper redditus inviso, me mihi tempusque auferunt, et efficiet Solodurensis Legatio ${ }^{12}$, ut vix Cels. Magistratui de meis mentio fieri possit.

Te vero Doctorem proxime salutare spero et cupio. Nova adcepi tam Lipsia a Schreibero ${ }^{13}$ quam Petropoli a Gmelino ${ }^{14}$. Schreiberus DoctorLegens est Lipsiae, Programma quo lectiones adnunciat, mihi remisit. Singulare admodum Medicinam definit «scientia eorum quae possibilia sunt per C. H. ${ }^{15}$ ), sic de caeteris. In cat(alogo) partium medicinae eliminat Hygienen et Semeioticen. Addidit novam edit(ionem) $\operatorname{Tr}$ (actatus) de fletu ${ }^{16}$ ubi varia problemata mathematice resoluta per aequationes simplices. Fastum quem ubique prae se fert excuso potius quam fero.

Ergo vere redeunte omni negotio conand. ut adcipiat plantas. Si velis, scribe ei per nuncium, plantas vero ad me mitte. Facile enim hinc omnia procurabo. GmeLInus post ... (Korrektur) recensionem, nuntiat se mihi remittere BuXBaumi Centurias II pl(antarum) minus cognitarum ${ }^{17}$ et alia. Acta v. Petropolitana jam adcepi. BuxBaumi fig(uras) vidi enim, nitidis-

11 Aus Gessners Brief vom 2.Dezember 1729 ist ersichtlich, daß es sich um die Manuscripta Bernoulliana, also die Nachschrift der mathematischen Vorlesung, handelte.

${ }^{12}$ Nach dem Berner Rats-Manual Nr. 124. (S. 145 ff.) hatte der französische Gesandte aus Anlaß der Geburt des Dauphins die eidgenössischen Stände zu einer Feier auf den 29. November 1729 nach Solothurn eingeladen. Da mit diesem Zusammentreffen auch politische Besprechungen verbunden waren («ratione der Reunion der Lobl. Eydtgenoßenschaft vndt wegen antragender Pundts Erneüwerung»), beschäftigte dieses «höchst wichtige Geschäft» den Rat der Zweihundert längere Zeit wegen der notwendigen Instruktion und der Beurteilung des Berichtes der Delegation.

13 Johann Friedrich Schreiber (1705-1760) war ein Studienkamerad HaLlers in Leiden.

${ }^{14}$ Johann Georg Gmelin (1709-1755), ein Studienfreund Hallers aus der Tübinger Zeit.

${ }^{15} \mathrm{Zu}$ lesen ist wohl: Corpus Humanum.

${ }^{16}$ Dissertatio de lacrymis et fletu, Lipsiae 1729, Bibl. anat. II, 218.

${ }^{17}$ J. Christian Buxbaum (1691-1730), Botaniker in Halle/S. Centurias II plantarum minus cognitarum, 1729, Bibl. bot. II, 170 . 
simae sunt, multa Tourneforti ${ }^{18}$ correxit, et nova addidit. Habentur etiam hic Inst(itutiones) Mathem(atices) in usus Imperatoris, ab HERMANNo et DE l'ISLE ${ }^{19}$. Praxin exercet amicus meus, et secat. Nundinae dederunt Bourgueti des Chrystaux ${ }^{20}$, ubi Belemniten, dentem ceti, lap. frumentarium etiam marini cujusdam reliquias pronunciat, curiosissima caeterum de mechanismo vegetabilium et animalium addens liber, quem si nondum habes, mittam Tibi, nec enim eo carere debes. Schurig, spurcissimus ille auctor de coitu muliebri amplum edidit volumen ${ }^{21}$. EGo v. TrewiJ orationem adcepi de praestantia anatomes ${ }^{22}$. Stahlius de vena aurea (haemorrh. $)^{23}$ germanice scripsit. Multa alia literar(ia) arctitudo excludit. Vale et me ut amasti, amare perge. Bernae 17. Nov. 1729.

Saluta omnes amicos Basilienses.

In der Sigerist (1923) zur Verfügung stehenden Sammlung befand sich nur ein einziger Brief Hallers an Gessner aus dem Jahre 1729, ebenso kannte VetTer (1909) ${ }^{24}$ keinen anderen aus dieser Zeit. Die beiden hier publizierten Briefe Hallers an Gessner aus dem Jahre 1729 haben also schon 1909 in der Sammlung gefehlt. Vetter wußte jedoch, daß weitere Briefe Hallers existiert haben müssen, da sich entsprechende Gegenschreiben Gessners in HaLlers Briefnachlaß vorfinden.

Es ist bekannt, daß Haller im Sommer 1729 zunächst nicht in Bern selbst, sondern auf dem Lande - wohl im Hasligut - gewohnt hat, wo er

18 Joseph Pitton de Tournefort (1656-1708), Professor der Botanik am Jardin des Plantes in Paris, publizierte nach einer Forschungsreise in den Orient: Corollarium institutionum rei herbariae, in quo plantae 1356 in Orientalibus regionibus observatae recensentur, Paris 1703.

19 Jacos Herrmann (1678-1733), zuletzt Professor der Mathematik in Petersburg, und Joseph Nicolas Delisle (1688-1768), Mitglied der Petersburger Akademie, publizierten gemeinsam: Abrégé des mathématiques à l'usage de S.M. Impériale de toutes les Russes, 3 vol. Petersbourg 1728 (PoggendorfF 1, 543 und 1077/8).

${ }^{20}$ Louis Bourguet (1678-1742), Professor der Philosophie und der Mathematik in Neuenburg: Lettres philosophiques sur la formation des sels et des cristaux, Amsterdam 1729.

${ }^{21}$ Martin Schurig, gestorben 1733 als Physikus in Dresden, publizierte: Parthenologia, Dresdae 1729, und Muliebra, morborum genitalium muliebrium consideratio, Dresdae 1729.

${ }^{22}$ Christoph Jacob Trew (1696-1769): Vertheidigung der Anatomie, Nürnberg 1729, Bibl. anat. $I I, 98$.

${ }^{23}$ Ernst Stahl (1660-1734): Abhandlung von der guldnen Ader, Leipzig 1729, Bibl. anat. I, 701.

${ }^{24}$ Ferdinand Vetter, Der junge Haller nach seinem Briefwechsel mit Johann Gessner aus den Jahren 1728-1738, Bern 1909. 
mit der Ordnung seiner Bibliothek beschäftigt war. Diese Arbeit hat sich offenbar bis weit in den Herbst hinein erstreckt, denn wir hören am 17. November, er sei erst kürzlich in die Stadt zurückgekehrt, verliere aber viel Zeit mit allerlei Kleinigkeiten. Insbesondere das Wiedersehen mit Freunden, Verwandten und Gönnern nähme seine Zeit in Anspruch. Der Auftrag zum Ankauf eines Pfundes Seide läßt allerdings auch die Möglichkeit offen, daß sich HALler vielleicht schon damals mit Heiratsplänen trug. Dazu kommt noch, daß Hallers Angelegenheiten den Berner Behörden wegen des wichtigen Geschäftes der Abordnung einer Gesandtschaft nach Solothurn nicht vorgebracht werden konnten. Wahrscheinlich bezieht sich dieser Satz auf die von Haller erhoffte Betätigung im Berner Inselspital, wo er seine wissenschaftlichen Arbeiten fortzusetzen gedachte. Bieten diese beiden Briefe Hallers auch nichts grundlegend Neues, so sichern sie doch unsere Kenntnisse gerade für die frühe, nur wenig bekannte Berner Zeit Hallers.

Wesentlich bedeutsamer ist der Inhalt des 3 . Briefes, der fast zwölf Jahre später geschrieben ist. HALLER hatte inzwischen seinen Weg fern der Heimat suchen müssen. Von Schicksalsschlägen verfolgt, bemühte er sich, durch ein Übermaß von Arbeit die ersten Jahre seiner Tätigkeit in Göttingen auszufüllen.

3. Brief, einzuschalten bei Sigerist (1923) zwischen Nr. 92 und 93.

Viro Celeberrimo/D(omino) D(octori) Joh. Gesnero P(rofessori) $\mathrm{P}(\mathrm{u}-$ blico) O(rdinario) / Can(onico) Carolino/S.p.d./Alb. Haller.

Adlata est tum litera Tua diu exoptata, tum diss(ertationum) fasciculus pro quo gratias, quas debeo Tibi ago, et He umanno ${ }^{21 a}$ nostro suam partem tradidi. Omnia gratissima fuerunt, sola mors fratris $\mathrm{Tui}^{25}$ tristis accidit, quem novi in eo stadio fuisse, per quod ad summam rerum humanarum abundantiam erat perventurus, totique familiae vestrae ornamento futurus. Sed ipse per quanta exempla didici, nihil nos possidere. Et nunc quoque, quoties expressam MARIANAE ${ }^{26}$ meae imaginem in filia contueor, ex priori jactura tristes facio conclusiones, forte et haec, quae Providentia mihi reliquit dilecta corpora, cito ex manubus debilis parentis et frustra retentantis a summo eventuum dispensatore erepta fore. Uti ereptus est dulcis

24a Christoph August Heumann (1681-1764), Professor in Göttingen.

${ }^{25}$ Gessner hatte im Brief vom 13. Juli 174.1 den am 10. Juli erfolgten Tod seines Bruders, des Stadtarztes und Dr. med. Christoph Gessner, gemeldet.

${ }^{26}$ Hallers erste Frau Mariane Wyss starb am 31. Oktober 1736 in Göttingen. 
filiolus BUCHERIAE ${ }^{27}$ meae, quantum videbatur, simillimus suavitate morum, vinculumque, quo ad familiam non ignobilem retinebar, unicum. Sed discite quaeso ex me, quid possimus pati. Exterus (!), aeger, inter ignotos mortales, hyeme nulla suppellectile expedita, amisi uxorem, qualem vidisti, aegrolantem habui puerum, nequidem alimenta facilia aut consueta tunc inveni. Diu me moeror, diu concepta corporis labes infirmavit, totius anni quotidianis purgantibus expuli abundans serum quo pedes tumebant et cachexia initia. Adfulsit spes novae beatitatis, uxor forma quidem quae cederet priori, ingenio, moribus gentis decore superior, et mei postquam me novit, amantissima ${ }^{28}$. Hanc ipsam crudelissimum puerperium me martyrium adspectante pene enecavit, deinde occulta uteri labes ex violento partu superstes absque ullo symptomate ex improviso, post immissum divinitus tenerae animulae horrendum tenorem eripuit. Puerum amabilem, blandissimum robustissimum jam dentatum, variolae confluentes occiderunt. Vivo in gente sui solius studiosa, de aliis unice secura, in collegio ubi non pauci impotentis animi homines et turbae intestinae cautos nos esse jubent et solitudinem quaerere. Vivo absque amico, qui Bernae lectissimis usus sum sodaliciis, vivo tamen, neque penitus illaetabilem vitam, quam amicae plantularum formae et exoptatissimi labores non sinunt esse adeo desperabilem, quam quidem eminus mente praesagiebam.

Ergo qui Te et morti et cumulorum invidiae eripuit, qui me magnis fractum malis erigit, idem porro et uxori defuncti fratris et caris parentibus eos inspirabit animos, qui ferre jacturam, et ex manu Parentis optimi bona malaque cum constante reventia accipere norint.

Gratissimum Tuum munus quaeso ne patere morari, non poteris ultra binos imperiales inpensae facere mitte per primam quaeso opportunitatem Norimbergam, aut Basileam denique, cum curric(ulo) publico, landkutschen, adventatum, aut si neque hoc tutum est, Bernam quaeso mitte ad Engelium $^{29}$ aut socerum Wyssium ${ }^{30}$. Cum enim exemplar ex Batavia recuperaverim, hodie in typographiam migrabit codex $^{31}$, quem loci Tui natales et plantae procul dubio, quibus egeo, aliquae misis modis ornabunt.

${ }^{27}$ Hallers zweite Frau Elisabeth Bucher, mit der er seit dem Juni 1739 verheiratet war, starb im Wochenbett am 4. Juli 1740; der damals geborene Sohn ist im Alter von sechs Monaten gleichfalls gestorben.

${ }^{28}$ Haller war seit dem Mai 1741 verlobt mit Sophie Amalia Christina Teichmeyer.

${ }^{29}$ Samuel Engel (1702-1784), ein Verwandter Hallers.

${ }^{30}$ Wahrscheinlich Samuel Wyss, HaLLERS Schwager, der 1755 starb.

31 Wohl die Enumeratio methodica stirpium Helveticarum, Gottingae 1742. 
Paratus erit ad a(nni) 1742 paschales nundinas et Tibi ipse aget gratias. Interim hac ipsa hebdomade prodit tomus III BoERHAAVIJ Tibi inscriptus ${ }^{32}$, quem opportuna aliqua occasione nata faciam, ut habeas.

Libri a CL. Оттіо ${ }^{33}$ gratissimi erunt. Synopseos meae pars maxima perfecta est. Superest trimestris fere labor. Fateor praestabile fuisse, si unam alterumque adhuc aestatem in alpinis et vulgarium characteribus expoliendis terere licuisset. Sed hanc moram res meae et Academicae, aegre concedebant, neque spes erat, cum remotus sim ab Alpibus multum me additurum. In Bructerum ${ }^{34}$ Sylvamque Hercyniam ineunte $\mathrm{m}(\mathrm{ense})$ Julio iter feci. Retuli characteres et plantas aliquas in priori non visas v. g. Genistum folio hyperici. Fungos etiamnum augerem, si liceret exspectare.

Cum ZUNDELIo ${ }^{35}$ serium sermonem habui. Incidit juvenis neque insulsus certe, neque indiligens, in manus cujusdam parasiti, MüLlERI, quem gratuitum contubernalem et nimis vereor, frequenter commensalem habuit. Vinum etiam hic limum, non, quod noverim nimis copiose, sed nimis contin(enter?) bibit, magnam itaque vim conflavit alieni aeris. Promisit Te scripturum, et Basileam iturum. Mihi videtur, perinde esse dum hinc discedab. ubi audio aliis etiam cum laqueis puellaribus retineri.

Nova literaria vix habeo. Nuper a Gerbero Petropoli semina accepi ${ }^{36}$, quorum aliqua mihi nihil nisi vulgatiorum videntur varietates. De LiNNAEO $^{37}$, Royenio ${ }^{38}$, Dillenio ${ }^{39}$ nihil quidquam. Heistero ${ }^{40}$ post filium, duo filiarum funera elata sunt. Anatomicum professionem Crellius ${ }^{41}$ qui-

${ }^{32}$ Der 3. Band von Hallers Werk: Hermanni Boerhave praelectiones, erschien 1741, er ist Gessner gewidmet.

${ }^{33}$ Vielleicht der Schaff hauser Arzt Јон. Отт, Bibl. bot. II, 31.

${ }^{34}$ Bructerum = der Brocken, mit 1142 Meter die höchste Erhebung im Harz (Sylva Hercynia).

${ }^{35}$ Nach Sigerist (1923), S. 132, Anm. 5, ein Schüler Gessners.

${ }^{36}$ Traugott Gerbers Brief vom 13. November 1740 ist aus Moskau geschrieben; er sandte u. a. ein Manuscript: Flora Moscuensis, durch das HALLER mit russischen, sibirischen und tartarischen Pflanzen bekannt wurde.

${ }^{37}$ Vgl. dazu ED. Fischer, Hallers Beziehungen zu den Naturforschern seiner Zeit, speziell zu Linné. Mitt. Naturf. Ges. Bern aus dem Jahre 1908, S. 145, Bern 1909.

${ }^{38}$ Adrian van Royen (1704-1778), mit Haller offenbar seit dessen Studium in Leiden bekannt.

${ }^{39}$ John James Dillenius (1687-1747), Professor der Botanik in Oxford.

${ }^{40}$ Lorenz Heister (1683-1758), Schüler Ruyschs, Professor der Chirurgie in Altdorf und Helmstedt, auch als Anatom bekannt.

${ }^{41}$ Johann Friedrich Creld, Professor in Wittenberg, später in Helmstedt. Haller führt in der Bibl. anat. II, 266 eine Reihe seiner Disputationes an. 
dam Wittebergensis replet. Lipsiae Platnerus ${ }^{42}$ pergit elaborare Chirurgiam, quae doctissima erit, est enim literatissimus, et libris instructissimus. In Anglia ediderunt BoerhaAviJ manuscriptum de calculo ${ }^{43}$ et editionem Inst(itutionum) cum tabulis aeneis ${ }^{44}$, nondum autem vidi.

Sed Tu vale precor, et fave, et quantocius fac habeam indicem ${ }^{45}$, herbas et Muraltum ${ }^{46}$, ut possim omnia in titulos distribuere. Saluta nobilem uxorem, parentes, fratrem. D. 1. Aug. 1741.

Bibliopola orat hanc schedulam tabellario publico tradas.

Der 3. Brief zeigt uns Haller in trüber Stimmung. Gessners Meldung vom Tode seines Bruders rief ihm die eigenen schmerzlichen Verluste in Erinnerung. Er gedenkt der beiden verstorbenen Gemahlinnen und des gleichfalls gestorbenen kleinen Sohnes der zweiten Frau. «Aber lernet bitte von mir, was wir erdulden können». Fern von den Freunden in der Fremde lebend, ist er nach dem Verluste der Gattin mit häuslichen Sorgen beladen. Er fühlt sich krank, sucht die Schwellung der Füße durch täglich genommene Abführmittel zu bessern und glaubt, Anfänge des Verfalles seiner Kräfte feststellen zu müssen. Mitten in all diesen Klagen über sein Mißgeschick und seine ihm unangenehme Umwelt findet sich aber plötzlich ein Satz, der von der Hoffnung auf kommendes Glück berichtet, eine erste, dem Freunde gemachte Andeutung neuer Liebe zu einer Frau. Es kann wohl keinem Zweifel unterliegen, daß es sich um Sophie Amalia Christina Teichmeyer handelt, die er noch vor Ablauf des Jahres 1741 geheiratet hat. Großes Gottvertrauen spricht aus den Zeilen des Trostes an seinen Freund. Von anderen persönlichen Angaben ist einzig noch interessant der Hinweis auf die zu Anfang Juli unternommene Reise in den Harz und auf den Brocken, die speziell botanischen Zwecken gedient hat.

42 Johann Zacharias Platner (1694-1747): Institutiones chirurgiae tum medicae tum manualis in usum discentium, Lipsiae 1745.

43 Boerhavii Libello de calculo, Londini 1741.

${ }^{44}$ Boerhavii Institutiones medicae in usus annuae exercitationis domesticos digestae. Cum tabulis aeneis recusae, Londini 1751.

${ }^{45}$ Index der Fundorte seltener Pflanzen aus Gessners Sammlung.

${ }^{46}$ Johannes von Muralt (1645-1733): Physicae specialis pars quarta seu Helvetiae Paradisus, Zürich 1710, Bibl. bot. I, 620; vgl. Gessners Brief vom 13. Juli 1741, Epist. II, 41. 
4. Brief, bei Sigerist (1923) einzuschalten zwischen Nr. 99 und 100.

Clarissimo Viro / D(omino) D(octori) Jон. Gesnero Canonico Carolino / S.p.d. / A. Haller.

Hodie eodem die quo hae literae, sed lentius, iter adgredior, Vobiscum futurus die Veneris proximo. Gillenum ${ }^{47}$ ibi et Schobingerum ${ }^{48}$ spero me reperturum. Die Solis Scaphusium petam, quem maximae et summe necessariae causae ita urgent, ut ne hic quidem ob plantarum causam vel unico pede ab urbe abfuerim. Id vero in tam praecipiti transitu supererit mihi solatio, quod receptus in collegium ducentum virorum, proxime nunc a patria absum, paucos post annos in eam, ut spero rediturus. Lectissimae conjugi me commendo, cui metuo, ne amicitia nostra sit oneri. Vale et me porro ut soles ama. Bernae die 12. Maj. 1745.

NB. HEGNERo ${ }^{49}$ numorum numeravi aliquantum ut iter posset absolvere, ad imperiales 25 , crucigeros 76 . Cum variis impensis valde adtriverim, id quod adtuleram, pecuniae, gratum esset, si in transitu ... quantillum etc. posset numerari. Reliquas quae ob lectiones supersunt nulla egent properatione.

Den 4. Brief schrieb Haller in glücklicher Stimmung. Er war auf dem Wege, Gessner und andere Freunde in Zürich zu besuchen; ferner kann er melden, daß er bei der Burgerbesatzung am 16. April 1745 zum Mitglied des Rates der Zweihundert gewählt worden ist. Ein lang gehegter Wunsch ging ihm damit in Erfüllung, bedeutete die Wahl doch, daß seiner Familie nun jederzeit die Heimat offen stand. Schon wird auch im Briefe die Möglichkeit erörtert, in wenigen Jahren nach Bern zurückzukehren. Auffällig ist an diesem Schreiben immerhin, daß Haller von seiner Wahl noch berichtet, trotzdem ihm Gessner bereits am 20. April 1745 seine Freude über diesen Erfolg ausgedrückt hatte.

5. Brief, bei Sigerist (1923) einzuschalten zwischen Nr. 103 und 104.

Egregio Viro / D(omino) D(octori) Joh. Gesnero S./D. Haller.

Fasciculum Tua benignissima cura coemtum dudum accepi ${ }^{50}$. BAKE-

47 Peter Giller (1703-1764), ein Leidener Studienfreund Hallers; vgl. Carl Wegelin, Briefe des St. Galler Stadtarztes Peter Giller an Albrecht von Haller, Gesnerus 7, 1. 1950.

${ }^{48}$ Johann Caspar Schobinger (1701-1763), seit 174.1 Stadtarzt in St. Gallen.

${ }^{49}$ Wahrscheinlich Hans Heinrich Hegner von Winterthur, ein Schüler Gessners.

${ }^{50}$ Nach Gessners Brief vom 16. Juni 1747: Arnauld Eloy Gautier D'Agosti, Essai d'anatomie en tableaux imprimés, etc. Paris 1746 fol. 
RUM $^{51}$ vero Tibi emtum nuper miserunt, quem faciam ut habeas, constat vero $^{52}$ Shill. sive ${ }^{52}$. Video me pridem ${ }^{52}$ flor. pro serico nuper vero $25.34 \mathrm{xr}$ in aere Tuo esse. Si potes a Hegnero $18 \mathrm{imp}$ (eriales) mihi debitos obtinere, compendi faceres mihi alicujus epistolae laborem. Si renuit aut moratur per $\mathbf{D}$ (ominum) de MathoD $^{53}$ mittam. Pro plantis Virginiis plurimum sum devinctus. De Societate ${ }^{54}$ gratulor Tibi, et semina quantum potero si placet libenter mittam, nunc vero literis aliquibus aut inventis vix possum inservire, in tam enormi cumulo laborum, quo obruor, et quem auxit Prorectoratus secundo mihi delatus.

Ampliss(imo) et magnifico Eschero ${ }^{55}$ velim plurimam salutam dicas nomine meo. Carmina mea prodibunt hac aestate ${ }^{56}$. De TrewiJ ${ }^{57}$-mirifico consilio - nihil inaudivi. Quidni edit Gesneriones pulcherrimas schedas. O si mihi contigissent!

Florae Germaniae tenuem prodromum edam inter annum ad opus ipsum nescio an vita mea sufficerit - certe Academica sufficere non potest.

Iconum I. II. III fasciculos mittam ${ }^{58}$. Deceret pretium non adicere, sed exemplaria aegre bibliopola concedit qui pauca imprimet. Faciam tamen ut modico pretio habeatis - 6 imperial nam aliis 3 ducatis solvitur. Tertius fasciculos (!) inter 14 dies prodibit.

Hoc toto tempore plurimus fui in dissectionibus angiologici maxime argumenti. Historiam vasorum inprimis phrenicorum minutissime scripsi ${ }^{58}$. A plantis undique requievi.

Nova nulla habeo magni momenti. Albinus pergit tabulas edere ${ }^{59}$. AdAMI

${ }^{51}$ HenRY BaKer, Employment for the microscope, London 1753.

${ }^{52}$ Die Zahlen fehlen im Brief.

${ }^{53}$ Ein Verwandter von Hallers erster Frau.

${ }^{54}$ Gessner war 1747 zusammen mit HaLler und anderen Mitglied der Kgl. Schwedischen Akademie der Wissenschaften geworden.

${ }^{55}$ Hans Caspar Escher (1678-1762), seit 1740 Bürgermeister in Zürich, «einer der einsichtigsten und aufgeklärtesten Staatsmänner der alten Schweiz» (HBLS 3, 76).

${ }^{56}$ Die 4. Auflage der HaLlersschen Gedichte erschien 1748 in Göttingen.

${ }^{57}$ C. Jаков Trew (vgl. Anm. 22), praktizierender Arzt in Nürnberg, Herausgeber des Commercium litterarium Noricum.

${ }^{58}$ Alb. Haller, Iconum anatomicarum partium corporis humani praecipuarum fasc. I (1743), II (1745), III (1747). Im 2. und 3. Teil sind speziell auch die Gefäße des Zwerchfells bearbeitet.

${ }^{69}$ Bernhard Siegrried Albinus (1697-1770), Hallers Lehrer der Anatomie in Leiden: Tabulae sceleti et musculorum corporis humani, Leiden 1747 fol. max. Tabulae septem uteri gravidi (ungefähr gleichzeitig erschienen). 
micrographia ${ }^{60}$ multo copiosior est quam Bakeri. Parsoni seminum historia $^{61}$ non magni momenti est. Pluris experimenta D. BRowne LANGrish ${ }^{62}$ in vivis brutis animalibus capta, cum dissolventib(us) calculi amylaceo lacte mistis in vesiculam inpulsa facta. Hic in Germania undique quiescunt eruditi nempe vivunt inter perpetuas lectiones, absque publico ingenii specimine solus Linnaeus laboriosissimus est, cujus plurimas diss(ertationes) nuperas accepi a $\mathrm{D}$ (omino) Rosen ${ }^{63}$.

Tu vero cum generosa uxore vale et mihi, ut soles, perge favere. D. 24 . Mart. 1747.

Wenige Hinweise genügen zur Erläuterung des 5. Briefes. HALLER versieht zum zweiten Male das Prorektorat der Universität Göttingen, das ihm eine Menge zusätzlicher Arbeit verursacht. Trotzdem kann er den Abschluß eingehender Untersuchungen über die Gefäßverzweigung speziell am Zwerchfell melden. In Deutschland seien im übrigen die Wissenschaftler ausschließlich mit Unterricht beschäftigt, einzig LINNE arbeite fleißig.

6. Brief, bei Sigerist (1923) einzuschalten zwischen Nr. 104 und 105.

Amplissimo et Reverendissimo Viro / D(omino) Johanni Gesnero / Canonico Carolino Phys(ices) Math(eseos) P(rofessori) / S. p. d. /A. Haller.

Valde gratas fuerunt literae Tuae, ex quibus didici non displicuisse Tibi meas icones. Nunc botanicas aliquas paro, fere Sibirici soli. Tabulas $\mathrm{D}$ (omini) GAUTIER accepi, eae parvi momenti sunt ${ }^{64}$, et ne umbra quidem Albinianarum $^{65}$.

Poteris ad Academiam Suecicam scribere, ut ego feci, et gratias agere et si placet, literas mihi transmittere. Res dudum confecta est, neque a nobis vel cogitatione ulla expetita, cum hactenus nemo nisi Suecus in eam Academiam receptus sit. Anglica Societas nunquam scribit sed Secretarius unice, et nonnunquam longo post tempore, mittit Catalogum typis impressum, cui novi membri nomen insertum est. Hamburgenses notitiam So-

\footnotetext{
${ }^{60}$ George Adams (1708-1773): Micrographia illustrata, London 1746.

${ }^{61}$ Jacob Parsons, De seminibus vegetabilium, earumque partibus minoribus etc. Philos. Transact. 1744.

62 Browne Langrish, Physical experiments upon brutes, London 1746.

${ }^{63}$ Nils Rosen (1706-1773) Professor der praktischen Medizin in Upsala, oder dessen Bruder Eberhard (1714-1796), Professor in Lund, ein früherer Schüler Hallers.

64 Über die Tafeln von GaUtier vgl. Anm. 50.

${ }_{65}$ Über die Tafeln von Albinus vgl. Anm. 59.
} 
cietatis vestrae ${ }^{66}$ exscripserunt ex nostris Gottingensibus literariis novis, quae dirigo.

Valetudo mea paulo rectior est, non adeo juverunt purgantia, quam millepedum succus ${ }^{67}$, supersunt tamen tumoris non levia vestigia. Serio cepi nunc de reditu in patriam cogitare. Unica porta est, si in Angliam commissarii nomine missus fuero a Republica, quod fieri posset a. 1750. Alioquin luculentam hic pensionem deserere non potui, nisi post multos annos, quando certior spes erit consequendae praefecturae. Ita a. 1754 in patriam redirem, si Deus vitam concesserit. Potero in ratione Tibi perfectius inservire, in loco constitutus. Fac, quaeso, ut apud Magnif(icum) Escherum et BlaARERUM $^{68}$ aliosque Tuos amicos, ut in conventibus et ubi aderit opportunitas mei meminisse meque proceribus nostris commendasse velint, nam haec praefectura votis liberis $200^{\text {rum }}$ obtinetur, neque a fortuna pendet. Urget me cura familiae, cum filia 15 annorum sit, filius 12 , valetudo infirmior, tristis inter alienigenos vita, et omnis solatii expers.

Nunc etiam tentabo an possim libros Tibi procurare, mitte quaeso catalogum desideratorum. Ego vicissim hunc addidi, quem Tibi commendo.

LUDWIGIUS ${ }^{69}$ optimus non suis sed alienis unice observationibus usus est. Sed viro bono nolo molestus esse. Orchides forte a. 1749 (quo Decanus ero) ea tractabo ratione ${ }^{70}$ qua ALLIA ${ }^{71}$ sed res valde longa est, maxime ob auctores evolvendos. Stirpes siccas Tibi passim colligam et servabo.

Halae nemo est, qui possit tabulas botanicas edere, quare mane quaeso in proposito et ede Tuas. Ego vix quidquam ultra molior praeter florae Germanicae prodromum. Quonam abierit DilleniJ collectio ignoro hactenus.

Morgagno fasciculum addo quibusdam nugis quas Tibi destinavi. Haec reddet D(ominus) Seelmatterius Tobiniensis.

Catalogos 2 libror(um) desideratorum adicit pro Italia et Parisiis. Ego

${ }^{66}$ Die Naturforschende Gesellschaft in Zürich war 1746 gegründet worden und hatte ihre erste Sitzung 1747 gehalten.

${ }^{67}$ Nach der Onomatologia medica, Ulm, Frankfurt und Leipzig 1772, zu der HaLLER ein Vorwort schrieb, wirkt ein Auszug aus Asseln harntreibend und dient «zur Eröffnung aller verstopften Eingeweide», auch wird der Gebrauch bei Wassersucht empfohlen.

68 Hans BlaArer (1685-1757), Stadthauptmann in Zürich und Präses der Bürgerbibliothek.

${ }^{69}$ Christian Gottlieb Ludwig (1709-1773), Professor der Botanik in Leipzig.

${ }^{70}$ Hallers Publikation erfolgte unter dem Titel: Orchidis genus constitutum in den Acta Helvetica erst 1760.

${ }^{71}$ Allii genus naturale constitutum, Gottingae 1745. 
in Anglia Tibi si placet imperare inserviam. Vale et me porro ama. D. 10. Juli 1747.

Plurimum vero jube valere nob(ilissimam) Tuam uxorem et amicos nostros BoDMerumque ${ }^{72}$.

Nur knapp vier Monate auf den vorangehenden folgt der 6. Brief. Gesundheitlich geht es HALLER nun etwas besser, doch glaubt er, keineswegs leicht zu nehmende Zeichen einer Geschwulst an sich konstatieren zu müssen. Ernsthaft denkt er an die Möglichkeit, in die Heimat zurückzukehren. Der einzige Ausweg sei, daß er vielleicht von der bernischen Regierung als Kommissar nach England abgeordnet werde zur Verwaltung der dort angelegten Staatsgelder, was im Jahre 1750 geschehen könnte. Anderenfalls wäre es ihm erst nach vielen Jahren möglich, seine auskömmliche Stellung in Göttingen aufzugeben, wenn nämlich gewissere Hoffnung vorhanden wäre, eine bernische Landvogtei zu erhalten. HALLER nimmt daher in Aussicht, 1754 nach Bern heimzureisen, bittet den Freund aber schon jetzt, er möge bei allen sich bietenden Gelegenheiten seinen Namen einflußreichen Gönnern in Erinnerung rufen, damit diese ihn in vornehmen Berner Kreisen empfehlen. Die Wahl zum Landvogt geschähe nämlich in freier Abstimmung des Rates der Zweihundert und hänge durchaus nicht nur vom Zufall ab. «Sorge um die Familie, unsichere Gesundheit und Mangel allen Zuspruches bei dem traurigen Leben unter Fremden» zwingen HaLler zu diesem Schritt. Wie aus diesen ausführlichen Angaben hervorgeht, war also die Heimkehr auf lange Sicht geplant.

7. Brief, bei Sigerist (1923) einzuschalten zwischen Nr. 192 und 193.

Quando quidem ex morbo satis vehemente convalesco, nolui Te, si periculum meum audivisti, finem ignorare. Incedi d. 31. Mart. in febrem erysipelanam, satis similem ejus, quam dudum Gottingae tuli, desquamata est facies, cum febre subinde satis acri. Cum tamen intermissionem aliquam et formam quotidianam perciperem, corticem die $10^{\circ}$ morbi sumsi et nunc absque febre vivo, cum viribus etiam et appetitu reconcilior nondum tamen lectum reliqui.

Quae Allionius ${ }^{73}$ mittet facile cum meis Geneva advenire possunt.

72 Über Hallers Beziehungen zu Bodmer berichtet ausführlich Ludwig Hirzel, Albrecht von Hallers Gedichte, Frauenfeld 1882.

${ }^{73}$ Carlo Allioni (1728-1804), Professor der Botanik in Turin: Oryctographiae Pedemontanae Specimen, Paris 1757. 
A Ludwigio diu nihil inaudivi. Ni molestum est 6 flor. cum 5 bz. pro eo ThомаnNo ${ }^{74}$ quaeso numera et in rationes refer, quas cum advenerit MiLLERUS, diluam. Monetae enim nostrae alioquin non valde facile conciliantur.

Brownium facile exspectavero.

Mittam proxime varia et inprimis disput(ationum) vol III. practica priora, tum diss(ertationes) Suecicas, si a bibliopego eas recuperavi, cui cum pessima multarum charta sit, eas tradidi. Adsunt in N(ova) L(iteraria) Gottingensia.

Filia major in praeparatione adhuc versatur. Nobilis puer Senatoris a Bonstetten felicissime inoculatus fuit ${ }^{75}$, et nunc hoc curandi genus invalescit. Etiam WerLHOFIUS ${ }^{76}$ administrationem eam curat.

Si placet Russelium ${ }^{77}$ fasciculo addo. Sat dignum librum.

A tribus septimanis $\alpha \mu o v \sigma o \varsigma$ nunc demum ad mea studia redeo. Hilliuss ${ }^{78}$ non placet, non satis plantarum gnarus.

RAMSPEKIUS ${ }^{79}$ propediem domi erit.

Physiologia haeret et tab(ulis) et ornamentis.

Vale humanissime GeSnere et me ut soles ama. Bern. 19. apr. 1757.

HaLle R

${ }^{4}$ Gessner an Haller (10. Mai 1758): non enim memini, qualem Thommannum in Tuis litteris velis.

75 Übereinstimmend mit dieser Angabe schrieb Victor von Bonstetten (1745-1832) in seinen Lebenserinnerungen (Paris 1832), S. 16: J'ai été le premier Bernois inoculé de la petite vérole; c'était, je crois, dans les années 1753, 1754 ou 1755 (richtigzustellen auf 1757). Le grand Haller avait décidé mon père à me faire subir cette opération alors très-effrayante pour les parens. On me mit au régime plusieurs semaines avant l'opération qui fut très-douloureuse; l'incision était profonde et la mêche du virus fut déposée dans la plaie. On me fit rester au lit jusqu'après l'éruption; puis on me fit garder la chambre ... Mes boutons n'étaient pas très-nombreux, ... on y venait cueillir du virus pour plusieurs de mes jeunes compatriotes, et je crois que c'est par moi que, grâce à HALLER, l'inoculation fut introduite à Berne.

${ }^{76}$ Paul Gottfried Werlhof (1699-1767), mit Haller eng befreundeter Arzt in Hannover.

77 Alexander Russed, Natural history of Aleppo, London 1756.

78 JoHn HILL (1716-1775), The British Herbal, London 1756.

79 Jakob Christoph Ramspeck, seit 1748 Professor in Basel, hatte einen ihm gewährten Studienurlaub nach Holland und England weit überschritten; vgl. Sigerist (1923), S. 271, Anm. 1 . 
Zehn Jahre nach dem 6 . ist der letzte der hier veröffentlichten Briefe Hallers an Gessner geschrieben; er ist unvollständig, denn nur das Schlußstück liegt vor. Die Hoffnung, eine Landvogtei zu erhalten, hatte sich nicht erfüllt. Halle R bekleidete nun die seinen Fähigkeiten gar nicht entsprechende Stellung eines Rathausammanns in Bern; als unterste Sprosse der Leiter zu einem Aufstieg in höhere Staatsämter hatte er sie seiner einflußreichen Professur in Göttingen vorgezogen. Wieder steht, wie so oft in Hallers Briefen, ein Krankheitsbericht voran. HALLER hatte eine fieberhafte Erkrankung - ähnlich den früher in Göttingen durchgemachten - überstanden, von der er sich allmählich erholte. Nach drei Wochen des Nichtstuns könne er endlich seine Studien wieder aufnehmen. Des weiteren meldet Haller, daß in Bern ein Knabe mit glücklichem Ausgang inokuliert, d. h. mit echtem Pockengift geimpft worden sei. Die Behandlungsmethode breite sich mehr und mehr aus, auch WerLHOF wende sie an. Mit diesem interessanten Hinweis auf neue Wege der Pockenbekämpfung schließt der Brief.

Zweifellos existieren noch weitere Schreiben Hallers an Johannes Gessner. So ist z. B. im Juli 1928 ein Brief vom 6. April 1751 im Auktionskatalog CXXXVI der Firma Henrici, Berlin, offeriert worden, dessen Ankauf der Stadtbibliothek Bern nicht gelungen ist. Auch Sigerist ${ }^{80}$ und VetTe $^{81}$ haben ja in ihren Publikationen schon gelegentlich auf fehlende Briefe hingewiesen. Es wäre wünschenswert, wenn diese wahrscheinlich verstreut in Autographensammlungen befindlichen Stücke bekannt gemacht würden, damit der Forschung wenigstens eine Reihe Halle Rscher Briefe möglichst vollständig zur Verfügung stünde. Derartige Publikationen anzuregen ist - außer den hier gebotenen neuen Angaben - ein Hauptzweck dieser Mitteilung.

${ }^{80}$ Sigerist (1923), S. 91, Anm. 1, das Jahr 1734 betreffend.

${ }^{81}$ Ferdinand Vetter, Der junge Haller, S. 6, Bern 1909. 